\title{
Defining the transcriptional redundancy of early bodywall muscle development in C. elegans: evidence for a unified theory of animal muscle development
}

\author{
Tetsunari Fukushige, ${ }^{1}$ Thomas M. Brodigan, ${ }^{1}$ Lawrence A. Schriefer, ${ }^{3}$ Robert H. Waterston, ${ }^{2}$ \\ and Michael Krause ${ }^{1,4}$ \\ ${ }^{1}$ Laboratory of Molecular Biology, National Institute of Diabetes and Digestive and Kidney Diseases, National Institutes of \\ Health, Bethesda, Maryland 20892, USA; ${ }^{2}$ Department of Genome Sciences, University of Washington, Seattle, Washington \\ 98195, USA; ${ }^{3}$ Department of Genetics, Washington University, St. Louis, Missouri 63108, USA
}

\begin{abstract}
Myogenic regulatory factors (MRFs) are required for mammalian skeletal myogenesis. In contrast, bodywall muscle is readily detectable in Caenorhabditis elegans embryos lacking activity of the lone MRF ortholog HLH-1, indicating that additional myogenic factors must function in the nematode. We find that two additional C. elegans proteins, UNC-120/SRF and HND-1/HAND, can convert naïve blastomeres to muscle when overproduced ectopically in the embryo. In addition, we have used genetic null mutants to demonstrate that both of these factors act in concert with HLH-1 to regulate myogenesis. Loss of all three factors results in embryos that lack detectable bodywall muscle differentiation, identifying this trio as a set that is both necessary and sufficient for bodywall myogenesis in C. elegans. In mammals, SRF and HAND play prominent roles in regulating smooth and cardiac muscle development. That $C$. elegans bodywall muscle development is dependent on transcription factors that are associated with all three types of mammalian muscle supports a theory that all animal muscle types are derived from a common ancestral contractile cell type.
\end{abstract}

[Keywords: Myogenesis; MyoD; SRF; HAND]

Supplemental material is avaliable at http://www.genesdev.org.

Received August 14, 2006; revised version accepted October 26, 2006.

Vertebrate skeletal muscle development is dependent on a well-defined transcriptional cascade. Critical in this cascade are the myogenic regulatory factors (MRFs) MyoD, Myf-5, MRF-4, and Myogenin, a set of four related basic helix-loop-helix (bHLH) transcription factors that play essential roles in muscle cell fate specification and differentiation (for reviews, see Charge and Rudnicki 2004; Tapscott 2005). These proteins act in concert with other transcription factors, such as MEF-2 family members, to promote expression of muscle-specific target genes. The temporal pattern of gene expression during skeletal muscle differentiation is orchestrated by the combinatorial action of these transcription factors and inputs from several cell signaling pathways that modulate their transcriptional activity. The progress in our understanding of skeletal myogenesis to date has been remarkable given the complexity of vertebrate systems that employ several multigene families encoding transcription factors acting in overlapping and partially re-

${ }^{4}$ Corresponding author.

E-MAIL mwkrause@helix.nih.gov; FAX (301) 496-0201.

Article published online ahead of print. Article and publication date are online at http://www.genesdev.org/cgi/doi/10.1101/gad.1481706. dundant pathways. However, this complexity has obscured the precise roles for many of these transcriptional regulators within the context of the developing animal.

Caenorhabditis elegans offers an opportunity to study the transcriptional control of myogenesis in a developmental system that provides anatomical and genomic simplicity relative to the vertebrates. The bodywall muscle of the nematode is functionally equivalent to vertebrate skeletal muscle and contains only 81 cells in the embryo (for review, see Moerman and Fire 1997). Moreover, each of the multigene transcription factor families implicated in vertebrate skeletal myogenesis is represented by a single gene in C. elegans. Therefore, the worm provides an opportunity to explore myogenesis at a relatively simple genetic and cellular level.

Studies of invertebrate myogenesis have revealed many apparent divergent features in the transcriptional regulation of the skeletal muscle developmental program compared with vertebrate systems. Whereas a subset of MRFs appears to be both necessary and sufficient for vertebrate skeletal myogenesis (Tapscott 2005), mutants lacking the single MRF-related factor in Drosophila (Nautilus) or C. elegans (HLH-1/CeMyoD) are 
still able to specify and differentiate striated muscle (Chen et al. 1992; Balagopalan et al. 2001). In addition, MEF-2 is a critical factor for striated muscle differentiation in Drosophila (Lilly et al. 1994), as it is in vertebrate tissue culture (Olson et al. 1995), but its loss in C. elegans has no known effect on bodywall muscle development (Dichoso et al. 2000). Given that the myogenic transcription factors are evolutionarily conserved in sequence, and most have been shown to be functional in heterologous systems, the developmental differences in the roles for these factors has been puzzling. More recent studies suggest these functional differences may be subtler than they at first appear. In C. elegans, HLH-1 has recently been shown to be a potent myogenic factor in vivo that is able to convert almost all early blastomeres to bodywall muscle-like cells when ectopically expressed (Fukushige and Krause 2005). These results highlight the functional similarity of the MRFs in regulating muscle development from worm to man, suggesting that the fundamental core mechanisms of myogenesis in these evolutionarily divergent systems are conserved.

Because bodywall muscle can form and differentiate in the absence of HLH-1 activity, a key issue in understanding $C$. elegans myogenesis has been the identification and characterization of additional myogenic factors. Previous studies have identified two transcription factors that might function with HLH-1 in bodywall muscle development. One is UNC-120, the single serum response factor (SRF)-related protein in C. elegans. First identified genetically in a screen for uncoordinated movement, we originally cloned the gene encoding UNC-120 and showed it was involved in bodywall muscle differentiation. Subsequent studies have also implicated UNC-120 in bodywall muscle development, although its myogenic role has yet to be directly tested (Dichoso et al. 2000; Baugh et al. 2005b). A second candidate bodywall myogenic factor is HND-1, the only C. elegans protein closely related to the vertebrate HAND family of bHLH factors. In vertebrates, the HAND factors are best known for their role in cardiac ventricle development (Cserjesi et al. 1995; Srivastava et al. 1995), although studies also demonstrate more widespread functions in embryonic and extraembryonic tissue development (for review, see Firulli 2003). In C. elegans, the hnd-1 gene was first identified as a regulator of somatic gonad precursor (SGP) cell development (Mathies et al. 2003). Interestingly, reporter gene studies suggested that hnd-1 was also expressed in early embryonic blastomeres, including those that exclusively give rise to bodywall muscle cells.

The possible roles of both UNC-120 and HND-1 in bodywall myogenesis in C. elegans have yet to be fully defined. To date, only a single allele of unc-120 has been identified, and molecular information suggests it is likely hypomorphic (see Supplemental Material). Interestingly, a synthetic lethal analysis using RNA interference (RNAi) and available mutants identified unc-120 interactions with both $h l h-1$ and hnd-1, suggesting these three factors might define a "muscle module" (Baugh et al. 2005b). However, bodywall muscle development was not assayed in this study, and genetic studies failed to find interactions between hlh-1 and hnd-1 mutants affecting bodywall myogenesis (Mathies et al. 2003). This suggested that the synthetic lethality observed between hlh-1 and hnd-1 in the RNAi screen may be due, in part or in whole, to defects in nonmuscle lineages.

To clarify the roles of these three factors in bodywall muscle development, we assayed the myogenic potential of each of them individually and characterized predicted molecular null mutants in each gene. We demonstrate here that, like HLH-1, UNC-120 and HND-1 can each convert naïve embryonic blastomeres to muscle-like cells when ectopically produced. Genetic interactions among deletion alleles of all three genes demonstrated an important, and partially redundant, role for each in bodywall muscle development. Furthermore, elimination of all three transcription factors blocks detectable bodywall muscle differentiation. Taken together, these results define these three transcription factors as a set that is both necessary and sufficient for proper bodywall muscle differentiation during embryogenesis. The dependence of $C$. elegans bodywall myogenesis on transcription factors that are required in vertebrates for skeletal, cardiac, and smooth muscle supports the theory that animal muscle types all diverged from a common ancestral contractile cell type.

\section{Results}

\section{Identifying transcription factors functioning in early bodywall muscle development}

We have previously shown that early embryonic blastomeres can be converted to bodywall muscle-like cells after ectopic expression of certain transcription factors under control of a heat-shock promoter (Fukushige and Krause 2005). HLH-1 (CeMyoD) is particularly potent in this assay and is able to convert nearly all somatic cells to a muscle-like fate when ectopically expressed within the first $3 \mathrm{~h}$ of embryonic development. Ectopic expression of PAL-1, a Caudal-related homeobox factor, can also convert blastomeres to a bodywall muscle-like fate in cells in which the TCF/LEF-like factor POP-1 has been down-regulated or is constitutively low or absent. We combined our ability to alter embryonic cell fates to muscle-like cells with whole-genome microarray analysis of gene expression to identify transcription factors likely functioning early in the bodywall muscle development program (see Materials and Methods).

Gene expression profiles for myogenesis induced by HLH-1 or PAL-1 in manipulated embryos were analyzed and compared using microarray analytical software programs (see Materials and Methods). A spot check of muscle structural genes showed that both muscle induction paradigms resulted in very robust expression of these genes within $4 \mathrm{~h}$ and peak expression at the terminal time point (Supplementary Table S1). We limited our focus to a list of 934 potential transcription factor-en- 
coding genes (Reece-Hoyes et al. 2005), of which 887 were represented by one or more probe sets on the Affymetrix expression arrays that were used. We considered of greatest interest those genes that were strongly induced by PAL-1 and/or that mimicked the pattern of hlh-1 gene expression and were present in both data sets. Fewer than 43 genes fulfilled these criteria in each myogenic induction paradigm (Supplementary Table S2).

Both unc-120 and hnd-1 were identified in our microarray analysis, bolstering previous data implicating these genes in bodywall muscle development. Expression of unc-120 was induced in either the PAL-1 or HLH-1 experiment, and its expression level sharply increased early on and then was maintained throughout the time course of these experiments, including the initiation of terminal differentiation. The expression of hnd-1 in our microarray experiments was strongly, and transiently, induced by PAL-1. However, no hnd-1 expression was induced by ectopic HLH-1 activity.

\section{Both UNC-120 and HND-1 are myogenic in embryonic blastomere conversion assays}

Early embryonic blastomeres are competent to respond to a variety of transcription factors that regulate cell fate (Fukushige et al. 1998; Zhu et al. 1998; Fukushige and Krause 2005). We tested the ability of UNC-120 or HND-1 to convert embryonic blastomeres to a bodywall muscle-like fate when the genes encoding these factors are ectopically expressed from a heat-shock promoter (see Materials and Methods). Monoclonal antibodies recognizing three different muscle proteins were used to monitor myogenesis: myosin heavy chain A (MHCA), myosin heavy chain B (MHCB), and PAT-3/Integrin (MH25) (Francis and Waterston 1985; Miller et al. 1986). All three of these muscle markers are easily detectable early in bodywall muscle differentiation (mid-embryogenesis), distinguishing them from many other muscle markers that only become reliably detectable later in embryogenesis.

Our initial trials tested the myogenic potential of UNC-120 or HND-1 alone in an otherwise wild-type background. We found that UNC-120 was weakly myogenic in this setting whereas HND-1 showed no obvious myogenic activity (Fig. 1A). These results are quite distinct from those using HLH-1 that resulted in robust activation of the bodywall muscle-like fate in nearly all somatic blastomeres (Fig. 1A; Fukushige and Krause 2005), suggesting that both UNC-120 and HND-1 were less potent myogenic factors on their own. We therefore tested each factor in two additional, more sensitive embryonic blastomere conversion assays. RNAi knockdown of skn-1 and pal-1 together blocks specification of the EMS, C, and D founder cells (Bowerman et al. 1992; Hunter and Kenyon 1996; Fukushige and Krause 2005). Ectopic expression of either unc-120 or hnd-1 in the skn1, pal-1 double-RNAi background resulted in widespread myogenesis as assayed by MHCA (Fig. 1B). Similar effects were seen when RNAi was used to knockdown both mex-1 and pop-1, which results in the anterior three-quarters of embryonic blastomeres adopting a gut cell fate while the posterior quarter becomes muscle (Fig. 1C; Mello et al. 1992; Schnabel et al. 1996; Fukushige and Krause 2005; Maduro et al. 2005). Taken together, these embryonic conversion assay results demonstrated that both UNC-120 and HND-1 were myogenic on their own, albeit weakly so in comparison with HLH-1.

It was possible that the ability of UNC-120 and HND-1 to convert blastomeres to a muscle-like fate was dependent on HLH-1. We explored this possibility two ways. First, we stained embryos in which either UNC120 or HND-1 had resulted in ectopic muscle-like cells with antibodies against $\mathrm{HLH}-1$; both factors induced ectopic hlh-1 expression (data not shown). Second, we crossed the strains in which the heat-shock promoter was driving unc-120 or hnd-1 expression into an hlh-1null mutant background. Because loss of hlh-1 is lethal (Chen et al. 1994), the hlh-1(gv44) deletion allele (see below) was genetically placed over a GFP-tagged balancer, and effects on progeny $(25 \%$ of which are hlh-1null homozygotes and GFP-negative) were quantified. Activation of UNC-120 in progeny of the balanced, heterozygous hlh-1(gv44) parental strain resulted in $23 \%$ $(n=140)$ of embryos with very faint and patchy MHCA staining (Fig. 1D), a pattern never observed in these experiments carried out in a wild-type background. We concluded that robust myogenesis induced by UNC-120 was dependent on HLH-1 activity. In contrast, the frequency and robustness of myogenesis after induction of ectopic HND-1 activity was no different when comparing the wild-type to the hlh-1(gv44) mutant background $(n=79)$ (Fig. 1C,D), demonstrating that HND-1 myogenic activity is not HLH-1-dependent.

\section{Identifying deletion alleles for unc-120 and hlh-1}

To test genetically the functions of hlh-1, unc-120, and hnd-1 in bodywall myogenesis, we wanted molecular null alleles of each gene. For hnd-1, a deletion null allele had been previously characterized (Mathies et al. 2003). Genetically defined nulls (all point mutants) have also been previously characterized for hlh-1 (Chen et al. 1992, 1994). However, the potency of HLH-1 in myogenic cell fate conversion (Fukushige and Krause 2005) led us to question if these previously characterized genetic null alleles might actually have residual molecular activity. Therefore, for hlh-1 we sought a deletion allele that would be a molecular null for activity.

We isolated a 1246-base-pair (bp) deletion allele (gv44) of hlh-1 from a randomly mutated pool of animals (Dernburg et al. 1998) that eliminates exons 2-5, including the entire bHLH coding region required for dimerization and DNA binding (Fig. 2A); we assumed this allele was a molecular null for HLH-1 activity. Phenotypically, the strain was identical to the genetically defined null allele hlh-1(cc450), resulting in homozygous animals that all died shortly after hatching with a Lumpy Dumpy morphology (Table 1). We were able to fully rescue the mu- 
A
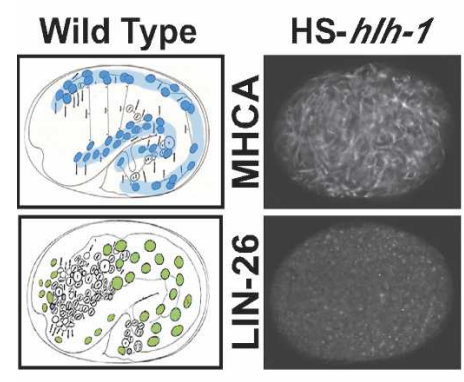

B

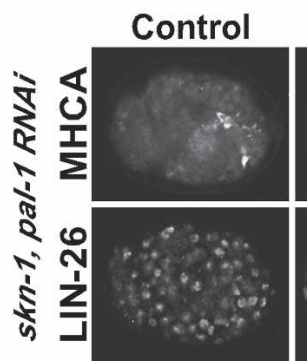

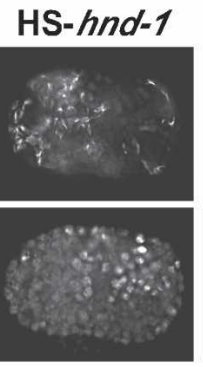

HS- $h$ nd-1

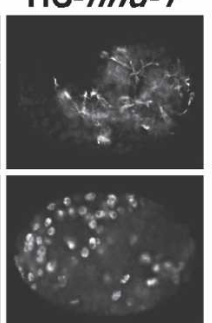

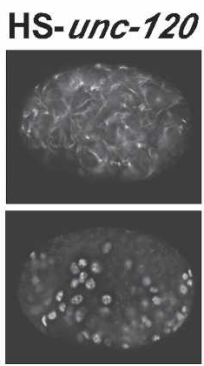

HS- unc-120

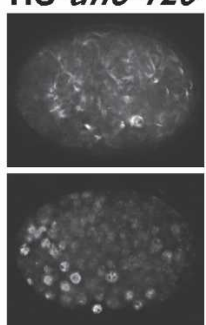

C

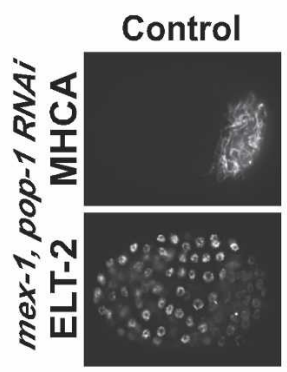

HS- $h$ nd-1
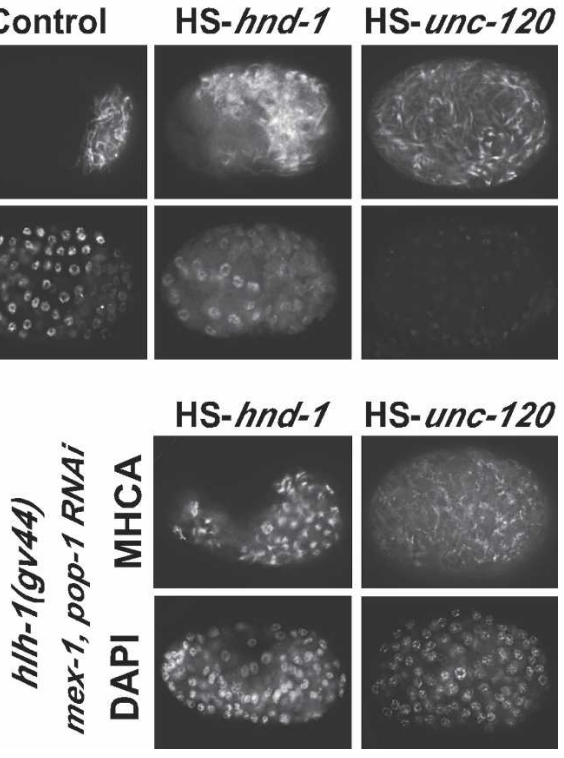

Figure 1. The myogenic potential of HLH-1, UNC-120, and HND-1. One of three transcription factors (as labeled) was induced in early embryos using the heat-shock promoter from integrated transgenic arrays. Terminally arrested embryos were stained with cell-type-specific antibodies to determine the extent of myogenic conversion. (A) Myogenic conversion in an otherwise wild-type background. HLH-1 has strong myogenic activity as indicated by robust MHCA staining (muscle marker) and the complete absence of LIN-26-positive cells (hypodermal marker). Both HND-1 and UNC-120 have relatively low levels of myogenic activity as neither can completely eliminate LIN-26-positive cells. Diagrams of wild-type embryos with expected staining patterns at mid-embryogenesis are shown at left for reference (adapted from Sulston et al. 1983, (C 1983, with permission from Elsevier). (B) Myogenic conversion in the skn-1, pal-1 double-RNAi background. Parental animals were treated with skn-1, pal-1 double RNAi to eliminate posterior embryonic cell fates in their progeny prior to induction of the specified transcription factor. The extent of bodywall muscle and hypodermis that develops after RNAi treatment alone is shown as the control. Both HND-1 and UNC-120 show increased myogenic activity compared with controls, although conversion in both cases was incomplete as indicated by numerous LIN-26-positive cells still present. $(C)$ Myogenic conversion in the mex-1, pop-1 double-RNAi background. Parental animals were treated with mex-1, pop-1 double-RNAi resulting in embryos that produce about three-quarters of the cells adopting ELT-2-positive gut cell fates (anterior) and one-quarter of cells adopting muscle cell fates (posterior) in control animals. Induction of either HND-1 or UNC-120 in the mex-1, pop-1 double-RNAi embryos resulted in gut-to-muscle transformations. HND-1 was only moderately effective at this as indicated by the large number of ELT-2-positive cells remaining after HND-1 induction. Muscle was detected in greater than half of the embryo volume in $30 \%-40 \%$ of embryos with ectopic HND-1. In comparison, UNC-120 shows robust myogenic conversion in this assay with widespread conversion of gut to muscle and a complete absence of ELT-2-positive cells. UNC-120 induced muscle in greater than half of the embryo volume in $80 \%-90 \%$ of embryos examined. (D) Myogenic conversion in the absence of hlh-1 gene activity. The ability of HND-1 and UNC-120 to convert gut cells to muscle in mex-1, pop-1 double-depleted embryos was tested in the hlh-1(gv44) mutant background (cf. results in C). HND-1 myogenic activity was indistinguishable in embryos with and without hlh-1 activity. UNC-120 retained myogenic activity in the hlh-1(gv44) mutant background, although MHCA expression was substantially reduced compared with embryos with wild-type levels of hlh-1 activity.

tant phenotype with a wild-type 7172-bp genomic fragment containing the hlh-1 gene. Subsequently, the Mitani C. elegans gene knockout resource kindly provided us with a second deletion allele [hlh-1(tm2090)]. As the phenotype and penetrance of the two deletion alleles were indistinguishable, we used the gv44 allele for all experiments.

The C. elegans arm of the National Bioresource Project of Japan, lead by Dr. S. Mitani, kindly provided us with the unc-120 deletion allele (tm1973) (Fig. 2B). This deletion allele removes 733 bp of genomic DNA beginning upstream of the start codon of unc-120 and extending to near the end of intron 1 . This deletion lacks the first exon that encodes most of the DNA-binding and dimerization domains of UNC-120. The absence of a start codon, and critical functional domains, led us to conclude that this allele is a molecular null and lacks all UNC-120 activity.

unc-120/SRF is required for robust bodywall muscle gene expression and interacts genetically with hlh-1

Homozygous unc-120(tm1973) mutants usually hatch (Table 1) and appear normal as early L1s. However, these animals become progressively paralyzed during L1 development and are $100 \%$ larval lethal, usually dying as late L1 or early L2 larvae (Supplementary Fig. S2C). We were able to fully rescue the mutant phenotype with a 7783bp wild-type genomic fragment containing the unc-120 gene. Although the lethality was rescued in animals car- 

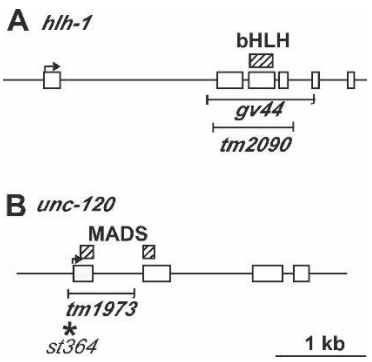

Figure 2. Schematic representation of mutant alleles. $(A)$ The hlh-1 gene structure is shown with exons as open boxes. Two deletion alleles (gv44 and tm2090) are shown below to indicate the regions missing for each. Note that both deletions include exon 3 encoding the bHLH domain (hatched box) required for dimerization and DNA binding. (B) The unc-120 gene structure with the deletion allele (tm1973) and point mutation allele st364 $\left(^{\star}\right)$ shown below. The deletion removes the start codon and most of the MADS-box region (hatched boxes) required for dimerization and DNA binding.

rying wild-type unc-120 genomic sequences on an extrachromosomal array, some rescued adult hermaphrodites were Egl. We interpreted these Egl animals as having lost the rescuing transgene in the egg-laying muscle lineages, suggesting that UNC-120 activity is required in these cells; this interpretation is consistent with results from the original hypomorphic allele (Supplemental Material). All phenotypic defects for unc-120(tm1973) were confined to bodywall and egg-laying muscle in hermaphrodites with no obvious perturbations of pharyngeal muscle or other cell types. Consistent with this, reporter gene expression driven by up to $4 \mathrm{~kb}$ of genomic sequence upstream of the predicted start codon of unc-120 showed strong expression that was limited to bodywall muscle cells and their precursors, beginning at $215 \mathrm{~min}$ of embryonic development (Supplementary Fig. S2), and in egg-laying muscles post-embryonically (data not shown).

Genetic interactions between unc-120 and hlh-1 were tested by making double-heterozygous mutants using balanced deletion alleles of each gene; only one in 16 $(6.25 \%)$ progeny of these double-heterozygous hermaphrodites are null for both UNC-120 and HLH-1 activity. However, we were able to identify homozygous hlh-1 mutant embryos visually by the absence of green fluorescent protein (GFP) expression because the hlh-1 mutant balancer includes three distinct promoter-driven GFP reporters. Whereas each mutant alone had very low levels of embryonic lethality, the double-heterozygous strain gave rise to $11 \%$ embryonic lethality among its progeny (Table 1). We stained synchronously staged progeny of the double-heterozygous strain at mid-embryogenesis (see Materials and Methods) with antibodies to MHCA, MHCB, MH25 (Francis and Waterston 1985), LIN-26 (a hypodermal cell marke) (Labouesse et al. 1996), and 3NB12 (a pharyngeal muscle marker) (Priess and Thomson 1987). All of the embryos were positive for all four markers. However, in $5 \%$ of the progeny from the hlh-1, unc-120 double-heterozygous parental strain we observed patchy MHCA staining with portions of bodywall muscle appearing absent (Fig. 3), a phenotype never observed in the single-mutant strains. The frequency of patchy staining is close to the expected frequency of double-homozygous mutant embryos $(6.25 \%)$. We concluded that unc-120 and hlh-1 mutants genetically interact and result in a more severe bodywall muscle phenotype than either mutant alone.

hnd-1 genetically interacts with both hlh-1 and unc-120

Previous studies of hnd-1 have described its expression and mutant phenotypes (Mathies et al. 2003). Reporter gene studies showed a very dynamic pattern of hnd-1

Table 1. Mutant lethality and MHCA-staining phenotypes

\begin{tabular}{|c|c|c|c|c|c|c|c|}
\hline \multirow[b]{2}{*}{ Strain } & \multicolumn{3}{|c|}{ Lethality } & \multicolumn{4}{|c|}{ MHCA staining } \\
\hline & $\mathrm{Emb}$ & $\mathrm{L}$ & $n$ & Wk & Pch & Neg & $n$ \\
\hline hlh-1 $\mathbf{1}^{\mathrm{a}}(\mathrm{cc} 450) /$ Bal1 & $4 \%$ & $21 \%$ & 902 & $6 \%$ & - & - & 172 \\
\hline hlh-1 $(g v 44) / B a l 1$ & $4 \%$ & $18 \%$ & 1277 & $12 \%$ & - & - & 171 \\
\hline unc-120/Bal 2 & $2 \%$ & $21 \%$ & 1828 & $21 \%$ & - & - & 218 \\
\hline hnd-1 & $6 \%$ & $11 \%$ & 1513 & $5 \%$ & - & - & 79 \\
\hline hlh-1/Bal1; unc-120/Bal 2 & $11 \%$ & $27 \%$ & 1470 & $18 \%$ & $5 \%$ & - & 81 \\
\hline hlh-1/Bal1; hnd-1 & $34 \%$ & $19 \%$ & 747 & $1 \%$ & $24 \%$ & - & 216 \\
\hline unc-120/Bal 2; hnd-1 & $22 \%$ & $12 \%$ & 3278 & $23 \%$ & - & - & 158 \\
\hline unc-120(Ex unc-120+);hnd-1 & $21 \%$ & $22 \%$ & 1392 & $44 \%$ & $19 \%$ & - & 137 \\
\hline hlh-1/Bal1; hnd-1; unc-120(RNAi) & N.D. & N.D. & N.D. & $43 \%$ & $28 \%$ & $11 \%$ & 164 \\
\hline hlh-1/Bal1; unc-120/Bal 2; hnd-1(RNAi) & N.D. & N.D. & N.D. & $17 \%$ & $14 \%$ & $10 \%$ & 62 \\
\hline unc-120/Bal 2; hnd-1; hlh-1(RNAi) & N.D. & N.D. & N.D. & $28 \%$ & $59 \%$ & $5 \%$ & 200 \\
\hline unc-120(Ex unc-120+);hnd-1; hlh-1(RNAi) & N.D. & N.D. & N.D. & $38 \%$ & $48 \%$ & $10 \%$ & 121 \\
\hline
\end{tabular}

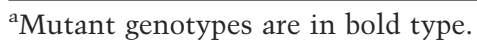

(Emb) embryonic; (L) larval; (n) $n$ value; (Wk) weak; (Pch) patchy; (Neg) negative; (N.D.) not determined; (RNAi) RNA-mediated interference; (hlh-1) hlh-1(gv44); (Bal1) mIn1[dpy-10(e128) mIs14]; (unc-120) unc-120(tm1973); (Bal2) lin-10(n1511) unc-29(e1072); (hnd-1) hnd-1(q740); (Ex unc-120+) a rescuing wild-type copy of unc-120 on an extrachromosomal array. 
Fukushige et al.

Figure 3. Myogenesis in single-, double-, and triplemutant embryos. Wild-type or mutant embryos, as indicated on the left, were stained at mid-embryogenesis for MHCA (muscle), LIN-26 (hypodermis), and DAPI (DNA). All images shown are lateral views with the anterior facing left. Images shown usually represent the most common mutant phenotype observed (see Table 1) unless otherwise indicated. At this stage of development, all single-mutant (hlh-1, unc-120, hnd1) animals show nearly normal levels of bodywall MHCA; an image of the unc-120 mutant is shown as representative of each. The severity of the bodywall muscle phenotype, as indicated by MHCA staining, is shown for each of the three double-mutant combinations; the unc-120, hnd-1 double mutant is from a parental animal carrying the mitotically unstable unc120 rescuing transgene; $44 \%$ of embryos have the phenotype shown. The hlh-1(gv44); unc-120(tm1973) double mutants show more cytoplasmically localized MHCA and patches devoid of staining, most obvious in the dorsal bodywall quadrants; $5 \%$ of all progeny from the double-heterozygous parent have the phenotype shown. The staining seen in the pharyngeal region of this embryo is due to costaining with the pharyngeal muscle marker antibody $3 \mathrm{NB} 12$ that was included as an additional control for permeability. The bodywall muscle phenotype is enhanced in the hlh1(gv44); hnd-1(q740) double mutants; $96 \%$ of all double-mutant progeny have the phenotype shown. Knockdown of unc-120 by RNAi injection of the hlh1(gv44)/mIn1; hnd-1(q740) mutant parental strain reveals the triple loss-of-function phenotype in the progeny embryos. MHCA is barely detectable on the ventral side in $50 \%$ of all triple mutants (shown) and completely undetectable in $\sim 25 \%$ of embryos with variability reflecting unc-120 RNAi efficiency. All triple-mutant combinations resulted in these same phenotypes, although the penetrance was dependent on the gene knocked down by RNAi (see Table 1). Note that in all mutant combinations, embryonic morphology appears relatively normal at this stage as indicated by LIN-26 and DAPI staining.
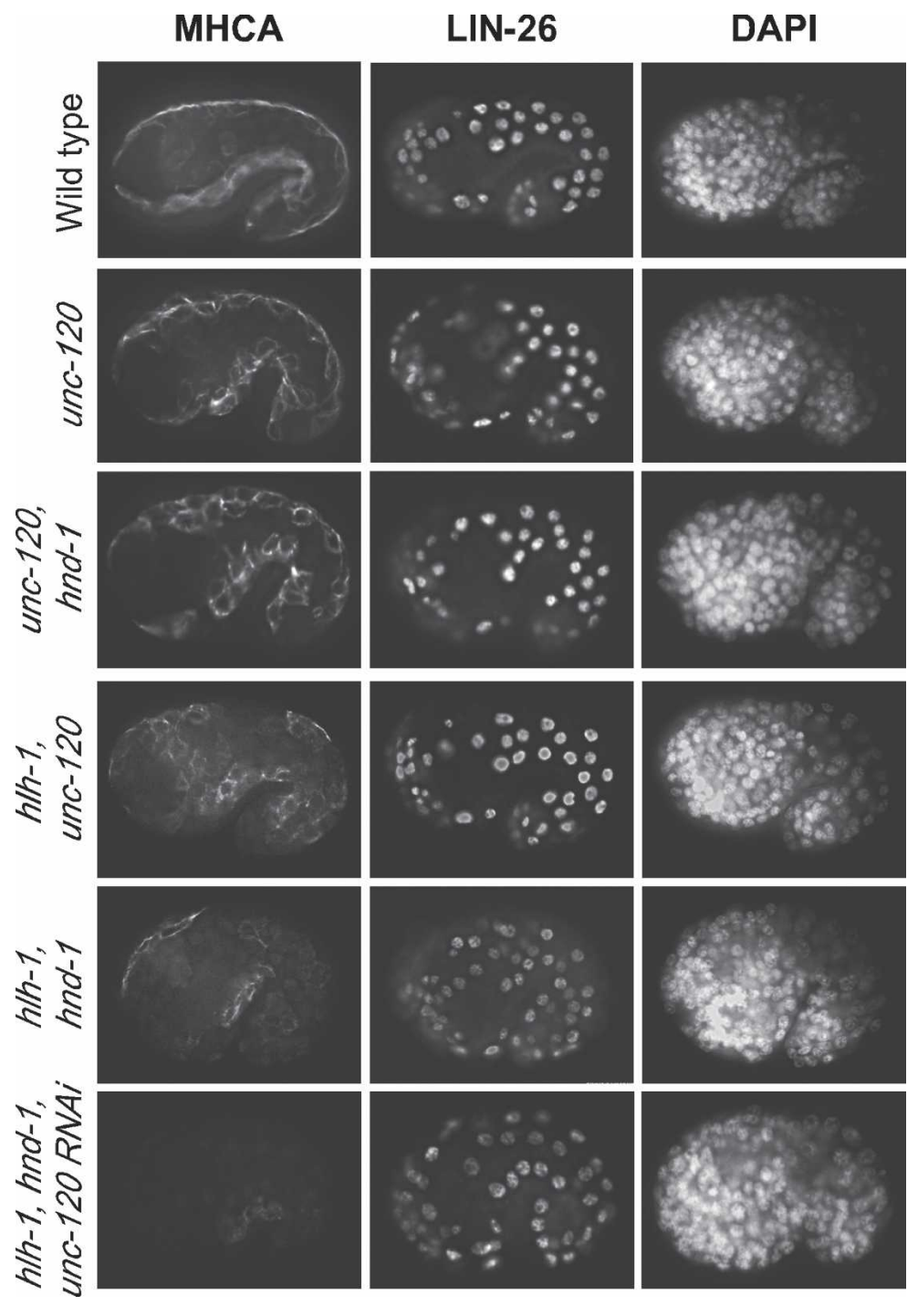

expression in early embryos, including transient expression in most, if not all, bodywall muscle lineages. We have obtained identical results with independent reporter genes, and we confirmed that HND-1 was transiently present in bodywall muscle lineages (and other lineages) using antibodies kindly provided by Laura Mathies (Department of Genetics, North Carolina State University, Raleigh, NC) (data not shown). The predicted null deletion allele hnd-1 (q740) is homozygous viable and fertile with a high frequency of somatic gonad defects due to perturbations in the development of the precursor cells Z1 and Z4 (Mathies et al. 2003). In addition, these animals occasionally had defects in embryonic development $(6 \%)$, often hatching with a phenotype reminiscent of homozygous hlh-1 mutants. Previously analyzed double-mutant combinations between hnd-1(q740) and the $h l h-1$ (cc450) point mutant failed to reveal any significant genetic interactions (Mathies et al. 2003).

We thought it was important to readdress the issue of genetic interactions between hnd-1 and hlh-1 using the new hlh-1 (gv44) deletion allele and direct assays of bodywall muscle differentiation. We constructed strains that were homozygous for the hnd-1(q740) deletion and heterozygous for the balanced $h l h-1$ (gv44) allele; $25 \%$ of the progeny of this strain are double-homozygous mutant. As shown in Table 1, 34\% of progeny from this strain die during embryogenesis. This compares with the singlehomozygous embryonic lethalities of $6 \%$ for hnd-1(q740) and $4 \%$ for $h l h-1$ (gv44). In addition to a threefold higher percentage of lethality than expected from a simple sum of the two mutants, embryonic defects were more severe than either mutant alone. Staining with MHCA and MH25 antibodies at mid-embryogenesis revealed that one-quarter of the embryos from $h l h-1(g v 44) / m I n 1$, hnd-1 (q740) parents had weak and patchy patterns (Table 1; Fig. 3).

We also tested genetic interactions between deletion alleles of hnd-1 and unc-120. Although hnd-1(q740) is homozygous viable, it was difficult to identify a strain harboring the homozygous hnd-1(q740) allele in the 
presence of even a single allele of unc-120(tm1973). Further investigation revealed that $90 \%$ of unc-120/+; hnd1(q740) hermaphrodites either lacked gonad arms or were sterile. To confirm that this was due to genetic interactions between these two genes, we used our unc120(tm1973) homozygous strain that had been rescued with a wild-type copy of unc-120 on an extrachromosomal transgene array. Although many animals are rescued by the transgene, the transgenic extrachromosomal array is mitotically unstable, and animals that lose the array die as L1s due to the loss of rescuing UNC120 activity. We were able to construct the unc120(tm1973), hnd-1(q740) double-homozygous mutant in the presence of the wild-type unc-120 rescuing array. However, $100 \%$ of the animals that lost the array (confirmed by PCR) were embryonic or early larval lethal. These results demonstrated that there is a very strong genetic interaction between hnd-1 and unc-120.

We assayed bodywall myogenesis in the unc120(tm1973), hnd-1(q740) double-mutant embryos derived from either unc-120/+; hnd-1(q740) parents or from rescued double-mutant parents that failed to segregate the rescuing extrachromosomal transgene to their progeny. The results from either parental genotype were identical; double-homozygous mutant progeny embryos had weak or patchy MHCA staining at mid-embryogenesis (Fig. 3). Staining these progeny with HLH-1 and MH25 antibodies demonstrated that bodywall muscle cells were present in the patchy MHCA staining regions (data not shown).

\section{Triple loss-of-function mutants fail to differentiate bodywall muscle}

Two factors made it difficult to study the triple homozygous hlh-1(gv44), unc-120(tm1973), hnd-1(q740) mutants genetically. First, the frequency of homozygous triple-mutant progeny derived from a triple-heterozygous and balanced hermaphrodite is extremely low, only one in $64(1.6 \%)$. Second, we could not take advantage of the viability of homozygous hnd-1(q740) animals in the presence of an unc-120(tm1973) allele because most of those animals are sterile (described above). For these reasons, we studied the triple loss-of-function mutants by combining genetic double mutants with RNAi knockdown of the third gene.

The most efficient of these knockdowns used the hnd1(q740), hlh-1(gv44)/mIn1 parental strain into which unc-120 double-stranded RNA (dsRNA) was injected. Twenty-five percent of the progeny of this parent are double homozygous for hlh-1(gv44), hnd-1(q740) and can be identified by their lack of GFP, and all should have reduced unc-120 activity. We found that $11 \%$ of the progeny in this experiment lacked all detectable MHCA staining when assayed at mid-embryogenesis (Table 1; Fig. 3). Triple-mutant embryos also lacked all MH25 staining in the bodywall muscle quadrants, although LIN-26 was normal (Fig. 4). As an independent confirmation of this result, we also injected $h l h-1\left(g_{V} 44\right) / m I n 1$, unc-120(tm1973)/lin-10 unc-29 hermaphrodites with dsRNA to knockdown hnd-1 expression. Ten percent of progeny from these animals failed to have detectable MHCA staining at mid-embryogenesis but were positive for the hypodermal marker LIN-26 (Table 1). This compares with the expected frequency of $6.25 \%$ for the double-homozygous deletion allele progeny from the balanced double-heterozygous parental strain. Finally, we injected hlh-1 dsRNA into the rare fertile unc-120/Bal2, hnd-1 mutant hermaphrodites; $5 \%$ of progeny embryos failed to stain for MHCA. A similar frequency $(6 \%-15 \%)$ of absent staining was obtained in triple-mutant/RNAi progeny stained with MHCA and MH25 together. We concluded that by multiple criteria, bodywall muscle differentiation could be completely eliminated in the triple-mutant embryos.

\section{Discussion}

This study has addressed a long-standing paradox in muscle development, namely the apparent divergent roles for the MRFs in regulating myogenesis in vertebrates versus invertebrates. Whereas the MRFs are essential for mouse skeletal muscle specification and differentiation, both C. elegans and Drosophila larval bodywall muscle are formed and differentiate in the absence of MRF activity. We show here that for C. elegans, this is due to the functions of two additional transcription factors: UNC-120/SRF and HND-1/HAND. Detectable bodywall myogenesis and near normal morphogenesis occur by mid-embryogenesis when one, or any two, of these factors are absent. However, loss of all three factors together eliminates all assayed markers of bodywall muscle differentiation. This study demonstrates that the core developmental program for bodywall muscle is evolutionarily conserved, a fact that was previously obscured by the partially redundant activity of these three transcription factors.

\section{UNC-120/SRF is critical for both early and late aspects of muscle differentiation}

Our work has demonstrated two important roles for UNC-120/SRF in C. elegans bodywall myogenesis. One is the ability of UNC-120 to initiate a bodywall muscle program in naïve early blastomeres or to transform gut to muscle. As previously described for HLH-1 (Fukushige and Krause 2005), the myogenic activity of UNC-120 can function cell autonomously, as demonstrated by the fact that often all somatic cells of the embryo are converted to muscle-like cells. Interestingly, robust (but not weak) UNC-120-directed myogenic conversion is dependent on HLH-1. Because hlh-1 regulates its own expression (Krause et al. 1994) and can activate unc-120, the robust myogenic potential of UNC-120/SRF relies on the hlh1-mediated positive autoregulatory feedback loop. A second role of UNC-120/SRF is to drive a high level of expression of many muscle structural genes, both in late embryogenesis and post-embryonic development. The phenotype of unc-120-null mutants, low-level myofilament protein production and post-embryonic lethality, is nearly identical to conditional mouse knockouts of SRF in skeletal muscle lineages that result in perinatal lethality due to skeletal muscle hypoplasia (Li et al. 
Figure 4. $\mathrm{MH} 25$ ( $\beta$-integrin) distribution in mutant embryos. The $\beta$-integrin marker recognized by $\mathrm{MH} 25$ was used to assess bodywall muscle formation in wildtype and mutant backgrounds as indicated to the left. All embryos were stained at mid-embryogenesis and LIN-26 (hypodermis) and DAPI (DNA) were used to verify embryo permeability and cellular organization. MH25 staining is slightly weaker in $5 \%$ of hnd1(q740) mutant embryos, as shown, but is noticeably weak and mislocalized in all hlh-1(gv44) mutants. MH25 is undetectable in bodywall muscle quadrants in $13 \%$ of triple-mutant embryos derived from unc120(tm1973)(Ex unc-120+); hnd-1(q740); hlh-1(RNAi) parents. These embryos reproducibly have diffuse cytoplasmic staining with MH25 in most cells of the embryo. These embryos also appear to have gut cells (based on nuclear size and shape by DAPI staining) displaced dorsally and fail to elongate beyond the comma stage even though wild-type and single-mutant embryos all reach at least the twofold stage.
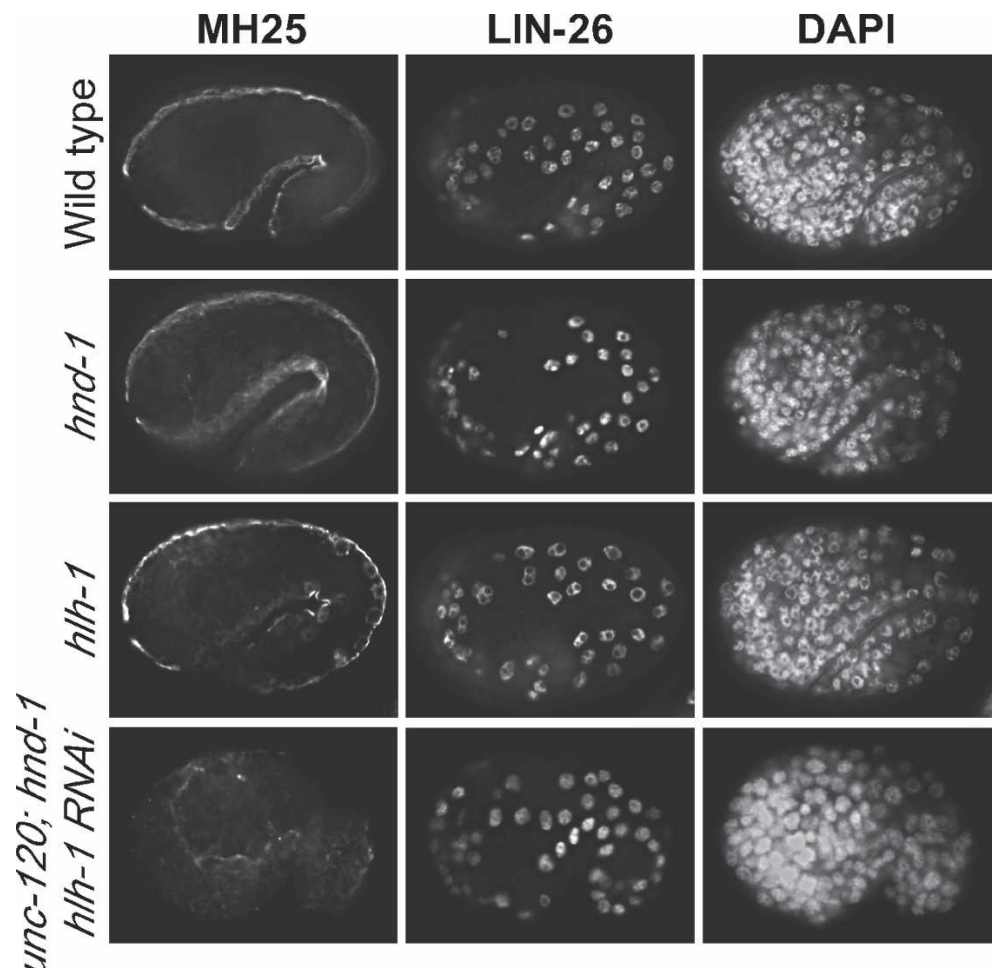

2005). Like its vertebrate counterparts, UNC-120/SRF functions to boost muscle gene expression in response to growth cues. UNC-120/SRF function is also critical for nonstriated egg-laying, but not pharyngeal, muscle development. UNC-120 function in post-embryonic nonstriated muscles is HLH-1-independent, suggesting it may interact with other factors, as does SRF in vertebrate cardiac and smooth muscle development. Vertebrate SRF-binding sites (CArG boxes) bind SRF in a complex with the cardiac and smooth-muscle-specific factor myocardin to regulate expression of genes required for differentiation (Wang et al. 2003; Niu et al. 2005). As there is no myocardin-related gene in C. elegans, other SRF-interacting partners are likely to exist. Importantly, our results demonstrate that the requirement of UNC$120 /$ SRF in muscle growth is evolutionarily conserved between $C$. elegans and mouse.

Our UNC-120/SRF results also explain another unusual feature of C. elegans myogenesis; that is, the lack of a requirement for MEF-2. MEF-2, like SRF, is a MADSbox transcription factor (Pollock and Treisman 1991). In vertebrates, MEF-2 acts with the MRFs to regulate skeletal muscle differentiation (Black and Olson 1998; Tapscott 2005). In contrast, C. elegans mef-2-null mutants are viable and phenotypically normal with no known bodywall muscle defects (Dichoso et al. 2000). We show here that this is because UNC-120/SRF acts with HLH-1 to drive myogenesis in the nematode, eliminating the need for MEF-2. Because UNC-120 is bodywall musclespecific during embryogenesis, and animals lacking MEF-2 appear normal, the nematode seems to lack the requirement for these MADS-box factors in regulating ubiquitous cellular responses to mitogens (Pollock and Treisman 1991).

\section{HND-1 provides transient redundancy in early} development

The role of HND-1 in bodywall muscle is distinct from that of UNC-120 and HLH-1. HND-1 is only transiently detected in the early bodywall muscle cell precursors, consistent with the description of hnd-1:gfp reporter genes (Mathies et al. 2003) and our microarray data; it is not detectable during muscle cell differentiation. In addition, HND-1 is present in many nonmuscle lineages where it also plays important developmental roles, such as somatic gonad precursor cell development post-embryonically (Mathies et al. 2003). In this sense, C. elegans HND-1/HAND is like its vertebrate counterparts that are required for the proper development of many different tissue types, in addition to roles in cardiac ventricle morphogenesis (for reviews, see Firulli 2003; McFadden et al. 2005). Our results are consistent with the general conclusion that HAND factors are critical for proper development, but not specification, of cell fates.

Our genetic results suggest that a critical role for HND-1/HAND in early C. elegans embryogenesis is to provide redundancy to $\mathrm{HLH}-1 / \mathrm{MRF}$ in activating muscle gene expression. The transient burst of HND-1/HAND expression in early embryonic bodywall muscle precursors is sufficient to act with UNC-120/SRF, resulting in transcriptional activation of UNC-120/SRF target genes. The ability of these two factors to interact to initiate much of the bodywall muscle program explains why muscle differentiation occurs in the absence of HLH-1 
(Chen et al. 1992, 1994). We have looked in hlh-1 mutants to see if HND-1/HAND persists until later stages of the bodywall muscle lineages; it does not. Thus, the transient burst of hnd-1 is sufficient, but not necessary, to augment the action of UNC-120 in muscle gene expression.

\section{A model for the transcriptional regulation of bodywall myogenesis}

We present a model in which HLH-1/MRF and UNC120/SRF represent the core factors required for bodywall muscle gene expression (Fig. 5), analogous to the vertebrate factors MRF and MEF-2. The primary role of HLH1/MRF is to maintain levels of UNC-120/SRF by initiating a positive autoregulatory feedback loop due to its own autoactivation and its ability to activate unc-120. In addition, HLH-1/MRF activates a subset of bodywall muscle genes that are distinct from those activated by the HLH-1/UNC-120 core transcriptional machinery. These are likely to be genes involved in cell-cell recognition given the disorganization of muscle quadrants and secondary hypodermal defects seen in hlh-1-null mutants. The role of HND-1/HAND is limited to early embryonic bodywall myogenesis where it functions to cooperate with UNC-120/SRF to initiate the onset of many muscle structural genes, principally providing redundancy to HLH-1/MRF function. It is likely that additional, as yet unidentified, transcription factors act in concert with the trio of myogenic regulators we describe here to fully orchestrate bodywall muscle development.

Our results validate previous suggestions that hlh-1, unc-120, and hnd-1 define a "muscle module." These three genes were shown to strongly interact in a systematic screen of genes involved in posterior embryonic development (Baugh et al. 2005a,b). This screen scored embryonic lethality but did not specifically assay muscle development. We now show, using novel null alleles of these genes in genetic combinations, specific bodywall muscle defects associated with loss of these factors, either alone or in combinations. We find in these studies that it is critical to use synchronously staged embryos assayed at mid-embryogenesis, near the beginning of bodywall muscle differentiation. In the absence of proper development, the bodywall muscle cells (and sometimes their neighbors) often undergo degeneration, making assays at terminal arrest misleading with respect to developmental roles. By carefully monitoring the onset of muscle differentiation, we have been able to tease apart the various roles for this trio of transcription factors.

Left unresolved in our model is the question of muscle cell fate specification in the triple-mutant embryos. The nearly normal arrangement of many other cell types at mid-embryogenesis (e.g., hypodermis) in animals lacking activity of all three factors suggests that cells from the multiple bodywall muscle lineages are born and migrate to the approximately correct position. This will need to be confirmed by direct lineage analysis through mid-embryogenesis once the technology exists to do this automatically, providing the throughput needed to be certain
A

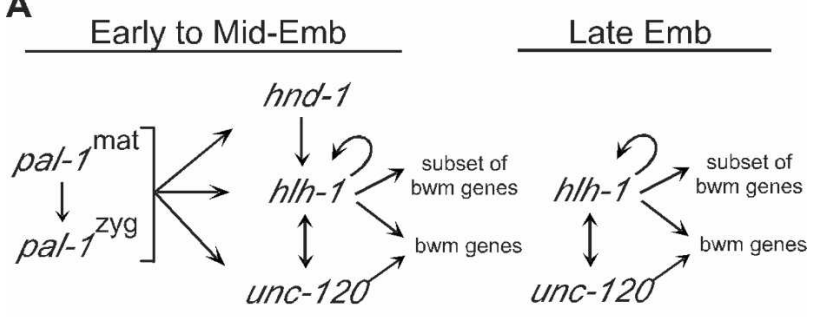

B

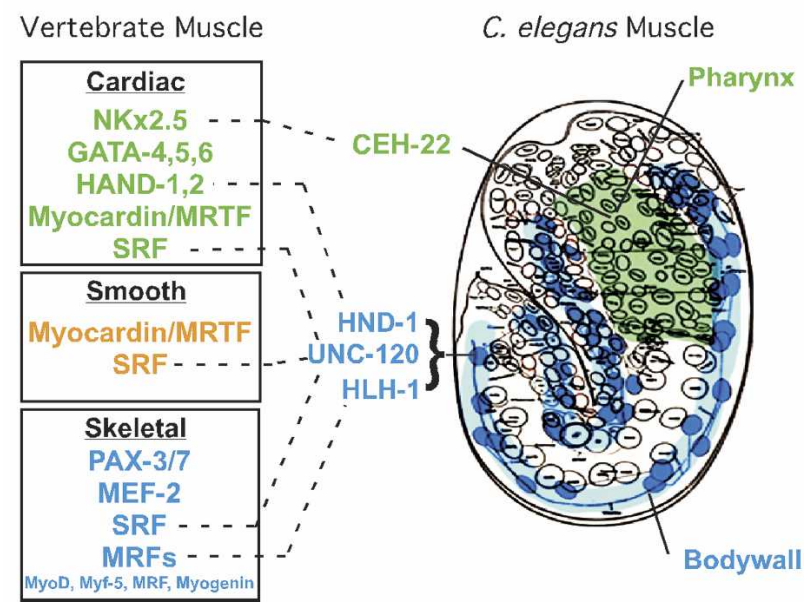

Figure 5. Transcriptional regulation of bodywall development. (A) Model for posterior blastomere bodywall myogenesis. In blastomeres from the posterior $\mathrm{C}$ and $\mathrm{D}$ lineages that have low or absent POP-1, maternal (mat) and/or zygotic (zyg) PAL-1 activates the bodywall muscle program via HLH-1, UNC-120, and HND-1. HLH-1 has several roles, including (1) to provide a positive autoregulatory feedback loop; (2) to activate and cooperate with UNC-120 to initiate muscle gene expression; and (3) to activate a small set of bodywall muscle-specific genes distinct from the set of HLH-1/UNC-120 targets. UNC-120 likely provides the key input for growth cues that boost muscle structural gene expression post-embryonically. HND-1 is partially redundant with HLH-1. Arrows reflect genetic results and do not necessarily indicate direct molecular targets. Embryonic bodywall muscle from the MS lineage uses a similar cascade but it is not triggered by PAL-1; the MS trigger is unknown. (B) Diagram of embryonic muscle (pharyngeal and bodywall) and the relationship of transcription factors that function in these C. elegans muscle types relative to the three major classes of vertebrate muscle (cardiac, smooth, skeletal). C. elegans embryonic bodywall muscle transcriptional regulators are related to factors that function in all three vertebrate muscle types.

of the relationship between phenotypes and genotypes. That information may change the question to one of defining what is meant by the term "bodywall muscle precursor cell." It is possible that cell fate specification is not limited to a small number of discrete and discernable characteristics, but, rather, it may be a continuous acquisition of cellular characteristics, punctuated presently by a limited set of molecular markers.

\section{Unified theory of muscle development}

An evolutionary question for debate is whether all muscle cells have a common contractile ancestor or if 
cells acquired contractility by independent evolutionary processes. An argument for independent evolutionary origins is based on the different protein isoforms, sarcomere organization, and contractile properties found in the three major classes of vertebrate muscle (skeletal, cardiac, smooth). In fact, MHC isoforms are defining characteristics that distinguish smooth versus striated muscle (for reviews, see Weiss and Leinwand 1996; Allen et al. 2000). Even within the striated musculature, skeletal and cardiac, there is clear evidence that regulatory transcriptional cascades are distinct and very ancient. Cardiac muscle transcription relies on Nkx-2.5, GATA4, GATA-5, GATA-6, and HAND-1 and HAND-2 factors (for reviews, see Schwartz and Olson 1999; Firulli 2003; Peterkin et al. 2005), whereas skeletal muscle requires MRFs and MEF-2 isoforms. Similarly, the two major muscle types in C. elegans, namely pharyngeal and bodywall, each utilize distinct MHCs (Miller et al. 1986) and distinct transcriptional regulators: the Nkx2.5-related factor CEH-22 for pharyngeal muscle (Okkema et al. 1997) and MRF-related factor HLH-1 for bodywall muscle (Krause 1995). Based on these characteristics, it can be argued that the last common ancestor of nematodes and mammals already had at least two distinct contractile cell types, one cardiac-like and one skeletallike (Fig. 5B).

Our results favor the alternative hypothesis, that cardiac, smooth, and skeletal muscle cells all had a common contractile ancestral cell type. We show here that C. elegans striated muscle development is dependent on the partially redundant action of $h l h-1$, hnd-1, and unc120. To date, the role of HND bHLH transcription factors in myogenesis has been limited to cardiac muscle. However, in C. elegans, hnd-1 is not expressed in the pharynx, nor is there a pharyngeal phenotype associated with the hnd-1(q740) deletion allele; its only known myogenic role is in striated bodywall muscle development. Perhaps more informative is the role of UNC-120/ SRF that is utilized for bodywall, but not pharyngeal, muscle development. In organisms from flies to mammals, SRF is a critical regulator of both smooth and cardiac muscle gene expression. Thus, in C. elegans bodywall muscle, transcription factors indicative of all three vertebrate muscle types are employed.

Evolutionary studies of muscle structural protein isoforms also suggest a common ancestral contractile cell type. Although orthologs of several vertebrate muscletype-specific structural protein isoforms can be traced to ascidians, there is remarkably little conservation in expression patterns relative to muscle type (Meedel and Hastings 1993). For example, structural protein isoforms indicative of vertebrate cardiac muscle are present in ascidian bodywall muscle and vice versa (Chiba et al. 2003), even though the ascidian heart is dependent on factors related to Nkx2.5 and HAND (Davidson and Levine 2003; Satou et al. 2004). Coupled with our results, a model emerges in which a single ancestral contractile cell was dependent on a transcriptional hierarchy comprised of factors we now associate with several different muscle types. Through speciation and muscle type spe- cialization, these transcription factors and their downstream target muscle structural genes may have become independently assorted in different phylogenetic branches to yield the muscle diversity we see today in animals.

\section{Materials and methods}

Strains

The strains used were wild type (N2); LGI: unc-120(st364), unc120(tm1973), lin-10(n1511)unc-29(e1072); LGII: hlh-1(cc450)/ mIn1[dpy-10(e128) mIs14], hlh-1(gv44)/mIn1/dpy-10(e128) mIs14] (Edgley and Riddle 2001), hlh-1(tm2090)/mIn1[dpy10(e128) mIs14], nDf4/lin-31(n301) bli-2(e768); LGX: hnd1(q740). Transgenic strains used were heat-shock pal-1(JA1179), heat-shock hlh-1 (KM267), heat-shock hnd-1 (KM403 and KM404), and heat-shock unc-120 (KM405 and KM406). hlh1(gv44) and unc-120(tm1973) mutants were rescued by PCRamplified genomic fragments from the respective wild-type genes using the primers ghlh-1F, 5'-GCGAGTTCTCGGTCT AACAGTGTCTCCCG-3'; ghlh-1R, 5'-CTAGCCTACCGCTG GAAAAATCTTCTGG-3'; unc-120p-F, 5'-AAGCTTAGTGTG CATTCCAACCATCTCTGTGCC-3'; unc120GR1, 5' -GAGTG TTGTCTGCTCCGCATTATCC-3'.

\section{DNA constructs}

The heat-shock unc-120 construct (pKM1236) utilized a fulllength unc-120 cDNA clone amplified from total mixed-stage RNA with the primers hs-unc120F (5'-GCTAGCATGACC GAAGCCGAAGACTTTGC- $\left.3^{\prime}\right)$ and hs-unc120R (5'-CCATG GTTATTCATTTGATGCAATGGCTGC-3'). The amplified cDNA fragments were digested with NheI and NcoI and inserted into NheI and NcoI sites of the pPD49.83 heat-shock promoter vector that was kindly provided by Dr. A. Fire (Department of Pathology and Genetics, Stanford University, Stanford, CA). The heat-shock hnd-1 construct (pJK902) was kindly provided by L. Mathies (Department of Genetics, North Carolina State University, Raleigh, NC) (Mathies et al. 2003). Both heat-shock unc-120 or hnd-1 transgenic animals were generated by microinjection of $100 \mu \mathrm{g}$ of pKM1236 or pJK902 and $50 \mu \mathrm{g}$ of pRF4 (dominant rol-6 as a transformation marker). The integrated heat-shock unc-120 or hnd-1 lines were generated by a $\gamma$-irradiation (Egan et al. 1995) and were back-crossed twice with the wild type (N2). Prior to heat shock, one- to two-cell stage transgenic embryos were isolated and incubated for $60 \mathrm{~min}$ at room temperature $\left(-22^{\circ} \mathrm{C}\right)$. Embryos were shocked for $30 \mathrm{~min}$ at $34^{\circ} \mathrm{C}$ and then incubated either $6 \mathrm{~h}$ or overnight at room temperature prior to fixation and antibody staining.

\section{Antibody staining}

Embryos were fixed with 5\% paraformadehyde on ice for 12 min, transferred onto a $0.1 \%$ gelatin-coated slide, and covered with a coverslip with slight pressure. Slides were frozen on dry ice for at least $30 \mathrm{~min}$, freeze-cracked, incubated in $100 \%$ methanol for $7 \mathrm{~min}$ at $-20^{\circ} \mathrm{C}$, dehydrated in PBS (phosphate buffer saline), and washed in $0.1 \%$ Tween 20, PBS for a least 15 min prior to incubation with primary antibodies. The primary antibodies used were chicken anti-HLH-1, rabbit anti-LIN-26 (kindly provided by Dr. M. Labouesse, Institut de Genetique et de Biologie Moleculaire el Cellulaire, C.U. de Strasbourg, Illkirch, France), monoclonal DM5.6 (MHCA) and monoclonal DM5.8 (MHCB) (kindly provided by D. Miller, Department of Cell and Developmental Biology, Vanderbilt University Medical Center, Nashville, TN), 3NB12 (pharyngeal muscle epitope), and MH25 (PAT-3/ß-integrin) (Developmental Studies Hybrid- 
oma Bank). A chicken anti-HLH-1 antibody was generated by following the vendor protocol (Sigma-Genosys) with an HLH-1 fusion protein (Krause et al. 1990) as antigen followed by affinity purification. The secondary antibodies (Jackson Immunological) used were rhodamine-conjugated donkey anti-rabbit IgG, fluorescein-conjugated goat anti-mouse IgG, and Cy3-conjugated donkey anti-chicken IgY.

\section{RNAi}

The full-length hlh-1 cDNA fragment from pVZ1200 (Krause et al. 1990) was inserted into the RNAi feeding vector L4440 (Timmons and Fire 1998). cDNAs for hnd-1 and unc-120 were amplified by RT-PCR using the primers listed below and were cloned into L4440. The RNAi constructs for mex-1, pop-1, skn1, and pal-1 were previously described (Fukushige and Krause 2005). The dsRNA products were checked by gel electrophoresis and were injected at $1 \mu \mathrm{g} / \mu \mathrm{L}$ for single-gene RNAi and/or 0.5 $\mu \mathrm{g} / \mu \mathrm{L}$ each for double-gene RNAi. The primers used for cloning cDNAs were unc-120F, 5'-ATGACCGAAGCCGAAGACTTT GCTC-3'; unc-120R, 5'-CTGCTGATTCGATGAGCTGTTGC AAG-3'; hnd-1F, 5'-ATGGTCAAATCAACAACTGCTGG-3'; and hnd-1R, 5' ${ }^{\prime}$-TTATTGCAAAATTGGATTACTGTTCTCC-3'.

\section{Microarray analysis}

Approximately 80 one- and two-cell stage embryos were collected from either heat-shock hlh-1 (KM267) or heat-shock pal1(JA1179) worms and were incubated for $20 \mathrm{~min}$ (hs pal-1) or 60 $\mathrm{min}$ (hs hlh-1) at room temperature prior to a heat pulse for 30 $\min$ at $34^{\circ} \mathrm{C}$. After the heat pulse, embryos were incubated for 2,4 , and $6 \mathrm{~h}$ (hs hlh-1) or 2, 4, 6, and $8 \mathrm{~h}$ (hs pal-1) at room temperature prior to total RNA isolation. Embryos were also collected prior to heat shock as a control $(0 \mathrm{~h})$. Triplicate independent samples were prepared for all time points. All hs pal-1 experiments were performed on embryos derived from skn-1, pop-1 double-RNAi-injected animals. The isolation of total RNA from embryos followed the procedures in Baugh et al. (2003). Total RNA was used with the Super Smart PCR cDNA synthesis kit (BD Clontech) in 20 cycles with a modified primer [SMART7T27 Primer, 5'-TGAAGCAGTGGTAACAACGCAG AGTAATACGACTCACTATAGGGAGAAGC $\left.(\mathrm{T})_{27} \mathrm{VN}-3^{\prime}\right]$ prior to a one-cycle target-labeling reaction by standard procedures (Affymetrix). Affymetrix C. elegans gene chips (P/N900383, $\sim 22,500$ transcript probes) were processed according to the manufacturer's protocol using the National Institute of Diabetes and Digestive and Kidney (NIDDK) Genomics Core Laboratory. Microarray data were normalized by MAS5 prior to analysis using GeneSpring software (Silicon Genetics).

\section{Acknowledgments}

We thank Robert Littlejohn for technical assistance, Shohei Mitani for kindly providing deletion alleles, David Miller III for MHC antibodies, Michele Labouesse for LIN-26 antibodies, Jim McGhee for ELT-2 antibodies, and Maggie Cam and George Poy of the NIDDK microarray core for processing samples. This research was supported by the intramural Research Program of the NIH, NIDDK. Some nematode strains used in this work were provided by the Caenorhabditis Genetics Center, which is funded by the NIH National Center for Research Resources (NCRR).

\section{References}

Allen, D.L., Harrison, B.C., and Leinwand, L.A. 2000. Inactivation of myosin heavy chain genes in the mouse: Diverse and unexpected phenotypes. Microsc. Res. Tech. 50: 492-499.
Balagopalan, L., Keller, C.A., and Abmayr, S.M. 2001. Loss-offunction mutations reveal that the Drosophila nautilus gene is not essential for embryonic myogenesis or viability. Dev. Biol. 231: 374-382.

Baugh, L.R., Hill, A.A., Slonim, D.K., Brown, E.L., and Hunter, C.P. 2003. Composition and dynamics of the Caenorhabditis elegans early embryonic transcriptome. Development 130: 889-900.

Baugh, L.R., Hill, A.A., Claggett, J.M., Hill-Harfe, K., Wen, J.C., Slonim, D.K., Brown, E.L., and Hunter, C.P. 2005a. The homeodomain protein PAL-1 specifies a lineage-specific regulatory network in the C. elegans embryo. Development 132: 1843-1854.

Baugh, L.R., Wen, J.C., Hill, A.A., Slonim, D.K., Brown, E.L., and Hunter, C.P. 2005b. Synthetic lethal analysis of Caenorhabditis elegans posterior embryonic patterning genes identifies conserved genetic interactions. Genome Biol. 6: R45.

Black, B.L. and Olson, E.N. 1998. Transcriptional control of muscle development by myocyte enhancer factor-2 (MEF2) proteins. Annu. Rev. Cell Dev. Biol. 14: 167-196.

Bowerman, B., Eaton, B.A., and Priess, J.R. 1992. skn-1, a maternally expressed gene required to specify the fate of ventral blastomeres in the early C. elegans embryo. Cell 68: 10611075.

Charge, S.B. and Rudnicki, M.A. 2004. Cellular and molecular regulation of muscle regeneration. Physiol. Rev. 84: 209238.

Chen, L., Krause, M., Draper, B., Weintraub, H., and Fire, A. 1992. Body-wall muscle formation in Caenorhabditis elegans embryos that lack the MyoD homolog hlh-1. Science 256: 240-243.

Chen, L., Krause, M., Sepanski, M., and Fire, A. 1994. The Caenorhabditis elegans MYOD homologue HLH-1 is essential for proper muscle function and complete morphogenesis. Development 120: 1631-1641.

Chiba, S., Awazu, S., Itoh, M., Chin-Bow, S.T., Satoh, N., Satou, Y., and Hastings, K.E. 2003. A genomewide survey of developmentally relevant genes in Ciona intestinalis. IX. Genes for muscle structural proteins. Dev. Genes Evol. 213: 291302.

Cserjesi, P., Brown, D., Lyons, G.E., and Olson, E.N. 1995. Expression of the novel basic helix-loop-helix gene eHAND in neural crest derivatives and extraembryonic membranes during mouse development. Dev. Biol. 170: 664-678.

Davidson, B. and Levine, M. 2003. Evolutionary origins of the vertebrate heart: Specification of the cardiac lineage in Ciona intestinalis. Proc. Natl. Acad. Sci. 100: 11469-11473.

Dernburg, A.F., McDonald, K., Moulder, G., Barstead, R., Dresser, M., and Villeneuve, A.M. 1998. Meiotic recombination in C. elegans initiates by a conserved mechanism and is dispensable for homologous chromosome synapsis. Cell 94: 387-398.

Dichoso, D., Brodigan, T., Chwoe, K.Y., Lee, J.S., Llacer, R., Park, M., Corsi, A.K., Kostas, S.A., Fire, A., Ahnn, J., et al. 2000. The MADS-box factor CeMEF2 is not essential for Caenorhabditis elegans myogenesis and development. Dev. Biol. 223: 431-440.

Edgley, M.L. and Riddle, D.L. 2001. LG II balancer chromosomes in Caenorhabditis elegans: $\mathrm{mT} 1(\mathrm{II} ; \mathrm{III})$ and the $\mathrm{mIn} 1$ set of dominantly and recessively marked inversions. Mol. Genet. Genomics 266: 385-395.

Egan, C.R., Chung, M.A., Allen, F.L., Heschl, M.F., Van Buskirk, C.L., and McGhee, J.D. 1995. A gut-to-pharynx/tail switch in embryonic expression of the Caenorhabditis elegans ges-1 gene centers on two GATA sequences. Dev. Biol. 170: 397- 
419

Firulli, A.B. 2003. A HANDful of questions: The molecular biology of the heart and neural crest derivatives (HAND)-subclass of basic helix-loop-helix transcription factors. Gene 312: 27-40.

Francis, G.R. and Waterston, R.H. 1985. Muscle organization in Caenorhabditis elegans: Localization of proteins implicated in thin filament attachment and I-band organization. J. Cell Biol. 101: 1532-1549.

Fukushige, T. and Krause, M. 2005. The myogenic potency of HLH-1 reveals wide-spread developmental plasticity in early C. elegans embryos. Development 132: 1795-1805.

Fukushige, T., Hawkins, M.G., and McGhee, J.D. 1998. The GATA-factor elt-2 is essential for formation of the Caenorhabditis elegans intestine. Dev. Biol. 198: 286-302.

Hunter, C.P. and Kenyon, C. 1996. Spatial and temporal controls target pal-1 blastomere-specification activity to a single blastomere lineage in C. elegans embryos. Cell 87: 217-226.

Krause, M. 1995. MyoD and myogenesis in C. elegans. Bioessays 17: 219-228.

Krause, M., Fire, A., Harrison, S.W., Priess, J., and Weintraub, H. 1990. CeMyoD accumulation defines the body wall muscle cell fate during C. elegans embryogenesis. Cell 63: 907-919.

Krause, M., Harrison, S.W., Xu, S.Q., Chen, L., and Fire, A. 1994. Elements regulating cell- and stage-specific expression of the C. elegans MyoD family homolog hlh-1. Dev. Biol. 166: 133148.

Labouesse, M., Hartwieg, E., and Horvitz, H.R. 1996. The Caenorhabditis elegans LIN-26 protein is required to specify and/or maintain all non-neuronal ectodermal cell fates. Development 122: 2579-2588.

Li, S., Czubryt, M.P., McAnally, J., Bassel-Duby, R., Richardson, J.A., Wiebel, F.F., Nordheim, A., and Olson, E.N. 2005. Requirement for serum response factor for skeletal muscle growth and maturation revealed by tissue-specific gene deletion in mice. Proc. Natl. Acad. Sci. 102: 1082-1087.

Lilly, B., Galewsky, S., Firulli, A.B., Schulz, R.A., and Olson, E.N. 1994. D-MEF2: A MADS box transcription factor expressed in differentiating mesoderm and muscle cell lineages during Drosophila embryogenesis. Proc. Nat1. Acad. Sci. 91: 5662-5666.

Maduro, M.F., Kasmir, J.J., Zhu, J., and Rothman, J.H. 2005. The Wnt effector POP-1 and the PAL-1/Caudal homeoprotein collaborate with SKN-1 to activate C. elegans endoderm development. Dev. Biol. 285: 510-523.

Mathies, L.D., Henderson, S.T., and Kimble, J. 2003. The C. elegans Hand gene controls embryogenesis and early gonadogenesis. Development 130: 2881-2892.

McFadden, D.G., Barbosa, A.C., Richardson, J.A., Schneider, M.D., Srivastava, D., and Olson, E.N. 2005. The Hand1 and Hand2 transcription factors regulate expansion of the embryonic cardiac ventricles in a gene dosage-dependent manner. Development 132: 189-201.

Meedel, T.H. and Hastings, K.E. 1993. Striated muscle-type tropomyosin in a chordate smooth muscle, ascidian bodywall muscle. J. Biol. Chem. 268: 6755-6764.

Mello, C.C., Draper, B.W., Krause, M., Weintraub, H., and Priess, J.R. 1992. The pie-1 and mex-1 genes and maternal control of blastomere identity in early $C$. elegans embryos. Cell 70: 163-176.

Miller, D.M., Stockdale, F.E., and Karn, J. 1986. Immunological identification of the genes encoding the four myosin heavy chain isoforms of Caenorhabditis elegans. Proc. Natl. Acad. Sci. 83: 2305-2309.

Moerman, D.G. and Fire, A. 1997. Muscle: Structure, function, and development. Cold Spring Harbor Press, Cold Spring
Harbor, NY

Niu, Z., Yu, W., Zhang, S.X., Barron, M., Belaguli, N.S. Schneider, M.D., Parmacek, M., Nordheim, A., and Schwartz, R.J. 2005. Conditional mutagenesis of the murine serum response factor gene blocks cardiogenesis and the transcription of downstream gene targets. J. Biol. Chem. 280: 32531-32538.

Okkema, P.G., Ha, E., Haun, C., Chen, W., and Fire, A. 1997. The Caenorhabditis elegans NK-2 homeobox gene ceh-22 activates pharyngeal muscle gene expression in combination with pha- 1 and is required for normal pharyngeal development. Development 124: 3965-3973.

Olson, E.N., Perry, M., and Schulz, R.A. 1995. Regulation of muscle differentiation by the MEF2 family of MADS box transcription factors. Dev. Biol. 172: 2-14.

Peterkin, T., Gibson, A., Loose, M., and Patient, R. 2005. The roles of GATA-4, -5 and -6 in vertebrate heart development. Semin. Cell Dev. Biol. 16: 83-94.

Pollock, R. and Treisman, R. 1991. Human SRF-related proteins: DNA-binding properties and potential regulatory targets. Genes \& Dev. 5: 2327-2341.

Priess, J.R. and Thomson, J.N. 1987. Cellular interactions in early C. elegans embryos. Cell 48: 241-250.

Reece-Hoyes, J.S., Deplancke, B., Shingles, J., Grove, C.A., Hope, I.A., and Walhout, A.J. 2005. A compendium of Caenorhabditis elegans regulatory transcription factors: A resource for mapping transcription regulatory networks. Genome Biol. 6: R110.

Satou, Y., Imai, K.S., and Satoh, N. 2004. The ascidian Mesp gene specifies heart precursor cells. Development 131: 25332541.

Schnabel, R., Weigner, C., Hutter, H., Feichtinger, R., and Schnabel, H. 1996. mex-1 and the general partitioning of cell fate in the early C. elegans embryo. Mech. Dev. 54: 133-147.

Schwartz, R.J. and Olson, E.N. 1999. Building the heart piece by piece: Modularity of cis-elements regulating Nkx2-5 transcription. Development 126: 4187-4192.

Srivastava, D., Cserjesi, P., and Olson, E.N. 1995. A subclass of bHLH proteins required for cardiac morphogenesis. Science 270: 1995-1999.

Sulston, J.E., Schierenberg, E., White, J.G., and Thomson, J.N. 1983. The embryonic cell lineage of the nematode Caenorhabditis elegans. Dev. Biol. 100: 64-119.

Tapscott, S.J. 2005. The circuitry of a master switch: Myod and the regulation of skeletal muscle gene transcription. Development 132: 2685-2695.

Timmons, L. and Fire, A. 1998. Specific interference by ingested dsRNA. Nature 395: 854.

Wang, Z., Wang, D.Z., Pipes, G.C., and Olson, E.N. 2003. Myocardin is a master regulator of smooth muscle gene expression. Proc. Natl. Acad. Sci. 100: 7129-7134.

Weiss, A. and Leinwand, L.A. 1996. The mammalian myosin heavy chain gene family. Annu. Rev. Cell Dev. Biol. 12: 417-439.

Zhu, J., Fukushige, T., McGhee, J.D., and Rothman, J.H. 1998 Reprogramming of early embryonic blastomeres into endodermal progenitors by a Caenorhabditis elegans GATA factor. Genes \& Dev. 12: 3809-3814. 


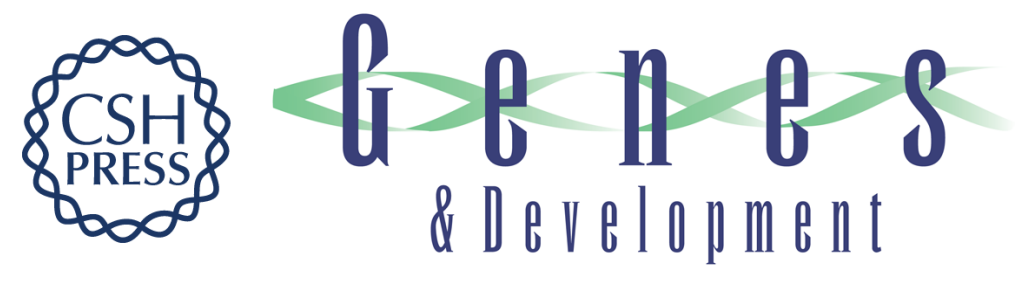

\section{Defining the transcriptional redundancy of early bodywall muscle development in C. elegans: evidence for a unified theory of animal muscle development}

Tetsunari Fukushige, Thomas M. Brodigan, Lawrence A. Schriefer, et al.

Genes Dev. 2006, 20: originally published online December 1, 2006

Access the most recent version at doi:10.1101/gad.1481706

\section{Supplemental http://genesdev.cshlp.org/content/suppl/2006/12/04/gad.1481706.DC1 Material}

Related Content

MyoD, modularity, and myogenesis: conservation of regulators and redundancy in C. elegans

L. Ryan Baugh and Craig P. Hunter

Genes Dev. December, 2006 20: 3342-3346

References This article cites 51 articles, 23 of which can be accessed free at:

http://genesdev.cshlp.org/content/20/24/3395.full.html\#ref-list-1

Articles cited in:

http://genesdev.cshlp.org/content/20/24/3395.full.html\#related-urls

\section{License}

Email Alerting

Service

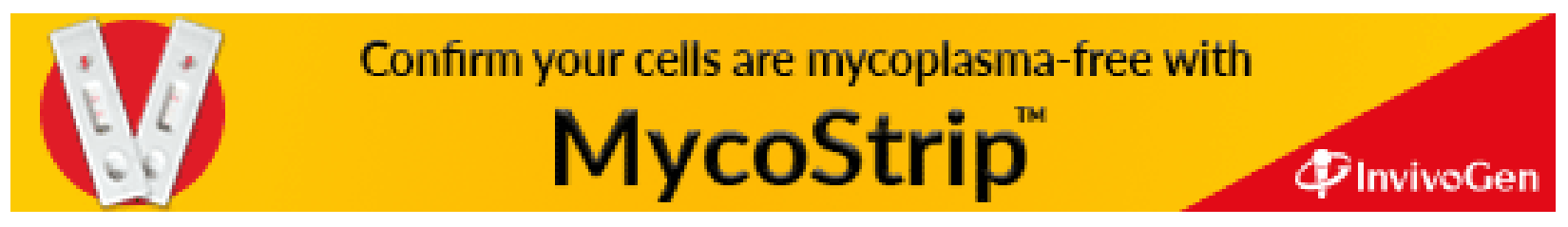

